\title{
Teenager als Manager ihrer Nahrungsmittelallergie
}

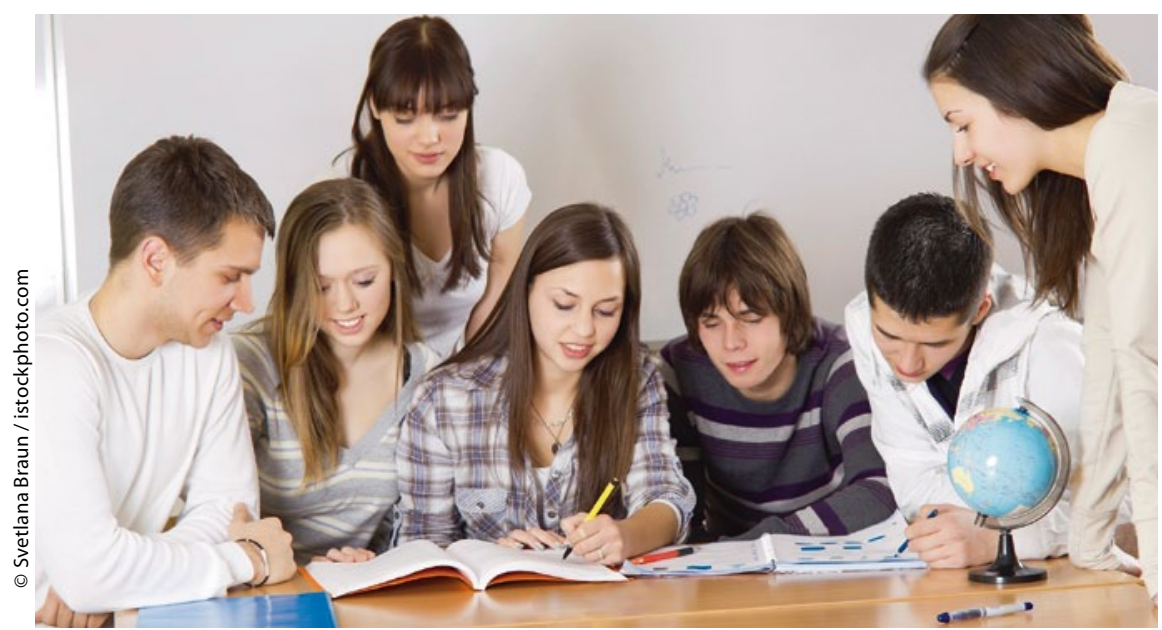

Ein eigenverantwortlicher Umgang mit ihrer Erkrankung kann die Compliance von Teenagern mit Nahrungsmittelallergie verbessern.

„Die Patientenschulung spielt eine Schlüsselrolle im Management von Nahrungsmittelallergien", betonte Prof. Dr. Anthony E. J. Dubois, University Medical Center Groningen, Niederlande. Doch die Frage sei, welche Inhalte sollten wem und wie vermittelt werden. Zu Schulungsprogrammen für Nahrungsmittelallergiker gibt es nur wenige wissenschaftliche Studien. Dubois stellte daher sein eigenes praktisches Vorgehen vor: Wird bei einem Kind eine Nahrungsmittelallergie diagnostiziert, hat die Familie viele Fragen: Welche Nahrungsmittel müssen künftig gemieden werden? Wie gefährlich kann die allergische Reaktion sein? Was tun im Notfall? An diesen Fragen orientieren sich die Inhalte, die mit dem Kind beziehungsweise den Eltern ausführlich besprochen werden müssen. Dabei sollte der Arzt unbedingt mit einer Ernährungsberaterin zusammenarbeiten. Bei Hinweisen, wie konsequent beispielsweise Spuren bestimmter Lebensmittel zu meiden sind oder wie vorsichtig man beim Essen im Restaurant sein sollte, müsse stets das mögliche Risiko und die Praktikabilität abgewogen werden, sagte Dubois. Wird für den Notfall ein Adrenalin-Autoinjektor verordnet, muss die Anwendung wiederholt geübt werden - auch damit das Kind merkt, dass dies wenig schmerzhaft ist, so Dubois.

Die Compliance von jugendlichen Betroffenen ist oft besonders schwierig zu erreichen. Meist motiviert es sie, wenn man ihnen vermitteln kann, dass sie selbst - und nicht die Eltern oder der Arzt - Manager ihrer Erkrankung sind. Teenager sollten darin gestärkt werden, selbst Verantwortung zu übernehmen und beispielsweise bestimmte Nahrungsmittel eigenständig zu meiden und den Autoinjektor stets bei sich zu führen und auch einzusetzen, empfahl Dubois.

Angelika Bauer-Delto

Dubois AEJ. Food allergy and anaphylaxis. Workshop 15 „ducating the child with allergies"

\section{Selbst "Spuren" von Nahrungsmittelallergenen meiden?}

Die Deklaration von Inhaltsstoffen in Lebensmittelprodukten gilt unter Allergologen als großer Fortschritt, um Nahrungsmittelallergiker vor bösen Überraschungen zu schützen. Zu großer Verunsicherung kann allerdings der Hinweis führen, dass "Spuren" bestimmter Substanzen enthalten sein können, kritisierte Prof. Dr. Bodo Niggemann, Charité, Berlin. Solche "Spuren" sind meist auf Kon- taminationen während des Verarbeitungsprozesses zurückzuführen. Es gibt jedoch keine einheitlichen Regelungen, was unter "Spuren“ zu verstehen ist und um welche Mengen es sich dabei handelt. Solche freiwilligen Warnhinweise werden von Hersteller zu Hersteller sehr unterschiedlich gehandhabt. Oft sind die Bezeichnungen der Substanzen auch für Laien kaum verständlich oder unleserlich klein auf der Verpackung aufgedruckt.

Beispielsweise auf Schokolade steht oft der Hinweis "Kann Spuren von Nüssen enthalten" - sollten Allergiker solche Produkte meiden oder nicht?
Lebensbedrohliche anaphylaktische Reaktionen auf solche "Spuren" seien nicht zu befürchten, meinte Niggemann. Ein Verzicht auf derart gekennzeichnete Nahrungsmittel schränke die Lebensqualität daher unnötig ein. Deshalb "Just relax" und den Hinweis einfach ignorieren, empfahl Niggemann provokativ. „Keineswegs!", konterte Kollegin Prof. Dr. Kirsten Beyer: Denn die Menge an Nahrungsmittelallergenen, auf die ein Patient reagiert lässt sich nicht exakt bestimmen und auch die Schwere der Reaktion sei nicht vorhersagbar. Patienten sollten daher auf Produkte verzichten, auch wenn nur "Spuren" an Inhaltsstoffen enthalten sind, auf die sie allergisch reagieren. Nahrungsmittel trotz eines solchen Hinweises zu verzehren, sei wie russisches Roulette, warnte Beyer.

Angelika Bauer-Delto

Pro \& Con „Should your patient avoid precautionary labeled food?" Beyer K. Pro. Niggemann B. Con. 